\title{
CROSS-COLOR BM3D FILTERING OF NOISY RAW DATA
}

\author{
Aram Danielyan ${ }^{1}$, Markku Vehviläinen ${ }^{2}$, \\ Alessandro Foi ${ }^{1}$, Vladimir Katkovnik ${ }^{1}$, and Karen Egiazarian ${ }^{1}$ \\ ${ }^{1}$ Department of Signal Processing, Tampere University of Technology, \\ P.O. Box 553, FIN-33101, Tampere, Finland. \\ web: www.cs.tut.fi/ lasip e-mail: firstname.lastname@tut.fi
}

${ }^{2}$ Nokia Research Center, Tampere, Finland. e-mail: firstname.lastname@nokia.com

\begin{abstract}
Color image reconstruction from noisy color filter array (CFA) data is considered. A modification of the Block Matching 3D (BM3D) [2] filter for CFA data denoising utilizing cross-color correlations is proposed. Denoised images are then demosaicked by algorithms developed for noise-free data leading to state-of-the-art performance for both Gaussian and Poissonian noise models.
\end{abstract}

\section{INTRODUCTION}

The common approach in single-chip digital cameras is to use a color filter array (CFA) to sample different spectral components. While various arrangements of the CFA have been suggested, the classical Bayer pattern [1] is still the mostly used nowadays. Full-resolution color components are reconstructed through demosaicking, i.e. the interpolation procedure estimating values of the two missing color components at each pixel. Independent interpolation of color channels usually leads to drastic color distortions, due to the aliasing introduced by subsampling. To compensate aliasing, demosaicking algorithms mainly exploit the high correlation between the red, green, and blue channels which is present in natural images. In particular, this means that the three color channels are likely to have similar texture and edge locations.

Most demosaicking algorithms have been developed under the assumption of noise-free data. In the presence of noise, the performance of the such algorithms degrades drastically. Three main strategies to deal with noisy data are possible: denoising after demosaicking, joint demosaicking-denoising (e.g., [5], [9], [10], [11]), and denoising before demosaicking (e.g., [7]). Denoising after demosaicking is very challenging, because sophisticated adaptive interpolation procedures change the statistical model of the noise in a complex and hardly computable form.

\footnotetext{
This work was supported by the Academy of Finland (project no. 213462, Finnish Programme for Centres of Excellence in Research 2006-2011, project no. 118312, Finland Distinguished Professor Programme 2007-2010, and project no. 129118, Postdoctoral Researcher's Project 2009-2011), by Tampere Graduate School in Information Science and Engineering (TISE), and by the Finnish Funding Agency for Technology and Innovation (Tekes, AVIPA3 Project).
}

Being deprived of an adequate noise model, the denoising step is quite ineffective, because it can rely essentially only on constraints reflecting the general a priori knowledge about the image structure. An attempt to make interpolation procedures robust against noise leads to the so-called "joint denoising and demosaicking" algorithms. Design of efficient joint algorithms is not an easy task because of the antagonistic nature of the denoising and interpolation procedures: denoising mainly performing some sort of data smoothing, while interpolation aims at reconstructing missing high-frequency details. The third approach, denoise and then demosaick, while apparently simple and straightforward, was long time considered to be inefficient [10],[9]. Direct application of conventional grayscale denoising filters to CFA is problematic due to the underlying mosaic structure of the CFA, which violates the basic assumptions about local smoothness in natural images which these filters rely upon. The standard approach is to split the CFA into four sub-images $\left(R, G_{1}, G_{2}\right.$, $\mathrm{B})$, filter these individually, and then recombine the filtered sub-images into a single denoised CFA image. The drawback is that subsampled images are aliased and filtering leads to the loss of many fine details and origination of artifacts when the denoised CFA image is demosaicked.

Nevertheless, modern denoising methods depart from the conventional local-smoothness constraints and instead they exploit non-local similarity of small image patches [6]. This development installs new potential for the "denoising before demosaicking" approach.

In [12], Zhang et al. propose a CFA denoising method based on the grayscale image filtering algorithm by Muresan and Parks [8]. This algorithm exploits the principal component analysis (PCA) for shrinkage on image blocks, where the covariance matrix used by the PCA is estimated from similar blocks found in a neighborhood.

In this paper, we develop and study the potential of cross-color filtering by applying the Block Matching 3-D filtering (BM3D) algorithm [2] directly in the CFA domain. This filtering is followed by a demosaicking step which assumes noiseless data. Extensive experiments with additive white Gaussian noise, as well as with signal-dependent Poissonian noise, demonstrate that our algorithm 


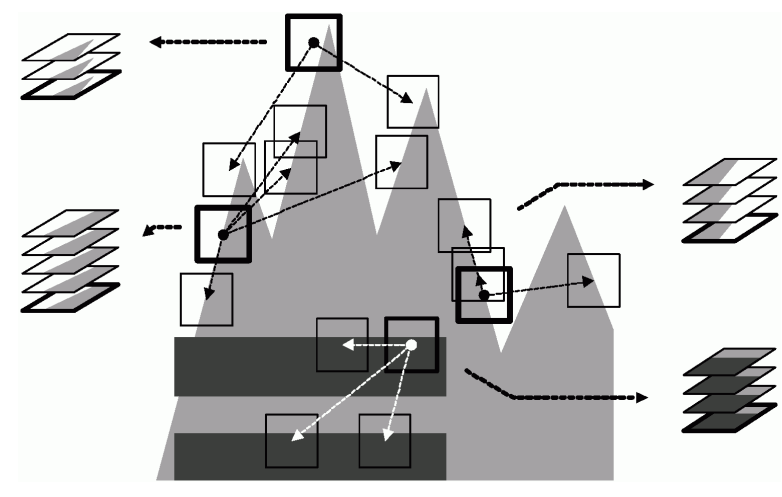

Figure 1. A simple example of grouping in an artificial image, where for each reference block (with thick borders) there exist perfectly similar ones.

works very well. In particular, it achieves numerical results which are uniformly better than the best state-ofthe-art results reported in [12]. The visual quality of the color images produced by our procedure is also very good, without the artifacts that typically arise when conventional filtering algorithms are directly applied to CFA prior to demosaicking. This ability of BM3D follows from the special non-local modeling embedded in this algorithm in order to reveal common features of similar image patches at different locations [6].

The rest of the paper is organized as follows. In the next section we discuss key principles of BM3D and introduce the proposed algorithm. Experimental results are given and discussed in the third section. Concluding remarks are made in the last section.

\section{BLOCK MATCHING AND 3-D FILTERING}

The general concept of the BM3D denoising algorithm is the following.

1. Block-wise estimates. For each block in the noisy image the filter performs:

(a) Grouping. Find blocks that are similar to the currently processed one, and then stack them together in a 3-D array (group).

The idea of the grouping is illustrated in Figure 1. Assuming that the stacked noisy blocks are corresponding to the perfectly identical noiseless blocks, an element-wise average (i.e. averaging between pixels at the same relative positions) will be an optimal estimator. In this way, we achieve an accuracy that cannot be obtained by processing the separate blocks independently. If the blocks stacked within the same group are not identical, averaging is no longer optimal. Therefore, a filtering strategy more effective than averaging should be employed.

(b) Collaborative filtering. Apply a 3-D transform to the formed group, attenuate the noise
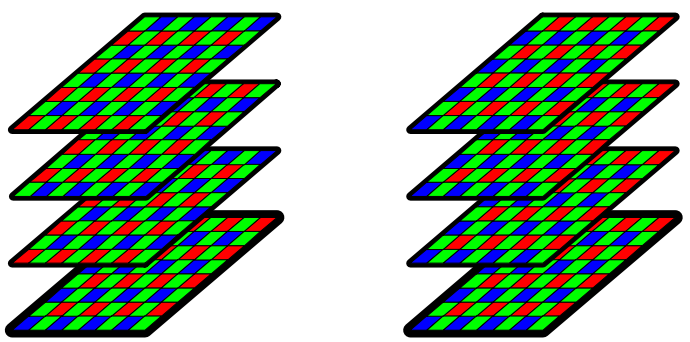

Figure 2. Illustration of a group composed of blocks having different color configurations (left) vs. one composed by blocks having the same color configuration (right).

by shrinkage (e.g., hard-thresholding) of the transform coefficients, invert the 3-D transform to produce estimates of all grouped blocks, and return the estimates of the blocks to their original place.

Due to the similarity between the grouped blocks, the transform can achieve a highly sparse representation of the true signal so that the noise can be well separated by shrinkage.

2. Aggregation. Compute the estimates of the output images by weighted averaging all of the obtained block-wise estimates that are overlapping.

A detailed description of the BM3D denoising algorithm can be found in [2]. Here, we intentionally give only general features of the BM3D algorithm referring to the paper [2], where the algorithm is introduced, and to the paper [6], where the evolution of the ideas exploited in this algorithm is discussed. One of the principal features of the BM3D algorithm is the so-called collaborative filtering enabled by the 3-D structure of groups formed from similar blocks. A multiple-model interpretation of this filtering is presented in [6].

\subsection{Color-constrained grouping}

Applying the original BM3D algorithm to CFA images can lead to severe checkerboard artefacts in regions with small (but non zero) intercolor difference. This happens when blocks with different color configuration are grouped and thresholded together, leading to a faulty intercolor difference estimation (for example, when a block with a red sample in its top-left corner is grouped with others having in their top-left corner a green or blue sample). This issue is easily and effectively resolved by restricting the grouping to blocks having the same color configuration, as illustrated in Figure 2. This is the only modification required in order to successfully apply the BM3D filter to noisy CFA data.

We remark that our filter does indeed exploit all color components simultaneously (hence the term cross-color filtering), because the shrinkage operates on the 3-D spectrum computed on a group which includes samples from all color components. 


\section{EXPERIMENTS AND DISCUSSION}

We evaluate the performance of the proposed denoising method by comparing it against the state-of-the-art PCAbased method [12] over three sets of experiments. First, assuming i.i.d. additive white Gaussian noise (AWGN) model, we consider the quality of the CFA images denoised by the two methods. Second, the denoised CFA images are demosaicked and compared. Third, we demonstrate results of denoising and demosaicking of images corrupted by the signal-dependent noise.

Throughout all experiments, four test images from the Kodak dataset are used: Flower, Houses, Lighthouse, and Parrots. Noise-free CFA images are obtained by subsampling the test images according to the Bayer pattern. All image intensities are assumed in the range $[0,1]$. The 3-D transform in the BM3D algorithm is realized as separable composition of 2-D discrete cosine (DCT) and 1-D Haar transforms.

\subsection{Additive white Gaussian noise}

First experiment. We compare the two denoising algorithms in two setups: a) filtering CFA as a single image and b) splitting the CFA into four color components, filtering them separately, and recombining back the denoised CFA image. Three different noise levels are considered $\sigma=\frac{5}{255}, \frac{12}{255}, \frac{25}{255}$. Numerical results (PSNR, dB) are presented in Table 1. Both algorithms show better results in denoising the CFA as a single image than componentwise, demonstrating their ability to take advantage of the cross-color filtering. In both setups, our algorithm achieves $0.8-1.9 \mathrm{~dB}$ over [12].

Second experiment. We interpolate the denoised CFA images from the first setup of the first experiment with one of the best demosaicking algorithms, the Directional Linear Minimum Mean Square-Error (DLMSEE) algorithm [13]. The numerical results summarized in Table 2 attest that the superior performance of our algorithm is largely maintained after demosaicking, with an advantage of about $1 \mathrm{~dB}$ over [12].

Visual inspection shows that CFA images denoised by the algorithm [12] suffer from strong residual noise, which also leads to color interpolation artefacts. Images denoised by the proposed algorithm, while looking noise-free, contain mild checkerboard artefacts in the areas with small intercolor difference. Some small details are better reconstructed by [12] thanks to the adaptivity of the PCA basis, compared to the fixed DCT transforms of BM3D. Zoomed parts of the corresponding images of Lighthouse are shown in Figures 3 and 4.

\subsection{Signal-dependent noise}

The noise in raw-data images from digital cameras is not i.i.d. Gaussian. It can be accurately modeled by the sum of a signal-dependent Poissonian component and a signalindependent Gaussian component and by taking into account the clipping due to the limited range of signal representation [3]. Let $y$ be the noise-free image and $x$ a pixel coordinate. The raw-data image $\tilde{z}$ can be expressed as [3]

$$
\tilde{z}(x)=\max \{0, \min \{1, y(x)+\sigma(y(x)) \xi(x)\}\},
$$

where $\xi(\cdot)$ is a random variable with zero mean and unit variance and $\sigma$ is a deterministic function of the form

$$
\sigma^{2}(y(x))=a y(x)+b
$$

with the constants $a \in \mathbb{R}^{+}$and $b \in \mathbb{R}$ depending on the sensor's specific characteristics and on the particular acquisition settings.

Most grayscale filters, including BM3D, are designed for i.i.d. Gaussian noise. Homomorphic nonlinear transformations can be employed for variance stabilization and for addressing the bias due to clipping [3], allowing us to utilize the algorithms BM3D and [12], designed for AWGN, for removal of the signal-dependent noise from the raw-data (1). For our simulations we set the noise parameters $a=0.004$ and $b=0.02^{2}$ (which reproduce the noise in the raw-data of a Fuji FinePix S9600 camera at ISO 1600), and rely on the variance-stabilizing and declipping transformations implemented in the ClipPoisGaus toolbox ${ }^{1}$.

The numerical results, presented in Table 3, are consistent with those for the AWGN case. The zoomed example in Figure 5 shows how the residual noise affect the quality of interpolation.

\section{CONCLUSION}

The recent development of powerful non-local denoising techniques, relaxing the local-smoothness constraints, opens possibilities to apply these techniques to CFA data directly. In this paper, we have shown how, after a slight modification, one of the best grayscale denoising methods, namely BM3D [2], can be effectively applied for direct CFA cross-color denoising. The denoised CFA can be then accurately demosaicked by algorithms developed for noiseless data. The experiments show a quite essential numerical and visual improvement pushing the stateof-the-art to a higher level. We have also shown that complementing this algorithm by variance-stabilization procedures enables its successful use for data with non-Gaussian signal-dependent noise.

\section{REFERENCES}

[1] Bayer, B.E., "Color imaging array," U.S. Patent 3 971065 , July 1976.

[2] Dabov, K., A. Foi, V. Katkovnik, and Egiazarian, K., "Image denoising by sparse 3D transform-domain collaborative filtering," IEEE Trans. Image Process., vol. 16, no. 8, pp. 2080-2095, Aug. 2007.

[3] Foi, A., "Clipped noisy images: heteroskedastic modeling and practical denoising", Signal Processing, vol. 89, no. 12, pp. 2609-2629, Dec. 2009.

[4] Hamilton, J.F., Jr., and J.E. Adams, “Adaptive color plane interpolation in single color electronic camera," U.S. Patent 5629 734, May 1997.

\footnotetext{
${ }^{1}$ http://www.cs.tut.fi/ foi/sensornoise.html
} 


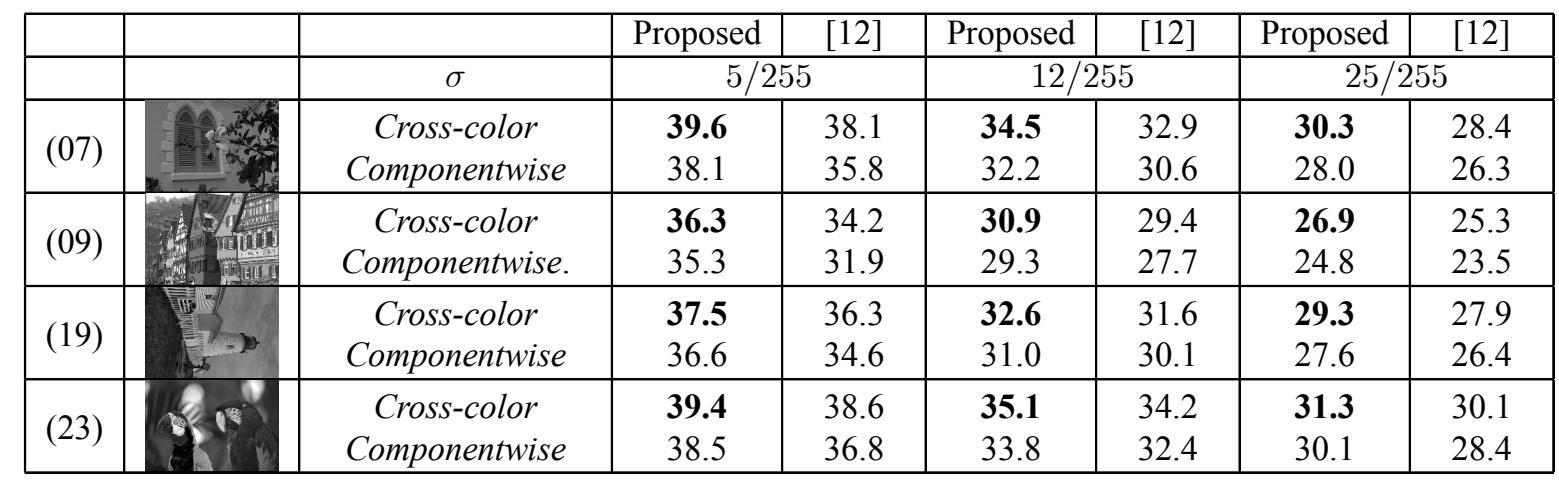

Table 1. PSNR (dB) results of the denoised CFA images of the first experiment. Computed excluding a 35-pixel border.

\begin{tabular}{|c|c|c|c|c|c|c|c|c|}
\hline & & & Proposed & {$[12]$} & Proposed & [12] & Proposed & [12] \\
\hline & \multicolumn{2}{|l|}{$\bar{\sigma}$} & \multicolumn{2}{|c|}{$5 / 255$} & \multicolumn{2}{|c|}{$12 / 255$} & \multicolumn{2}{|c|}{$25 / 255$} \\
\hline \multirow{3}{*}{ (07) } & & $\mathrm{R}$ & 37.8 & 36.8 & 33.9 & 32.6 & 30.1 & 28.5 \\
\hline & & $\mathrm{G}$ & 39.1 & 38.0 & 34.6 & 33.2 & 30.6 & 28.8 \\
\hline & & B & 37.5 & 36.6 & 33.8 & 32.6 & 30.1 & 28.3 \\
\hline \multirow{3}{*}{ (08) } & & $\mathrm{R}$ & 32.7 & 31.7 & 29.6 & 28.5 & 26.3 & 24.9 \\
\hline & & $\mathrm{G}$ & 34.5 & 33.2 & 30.5 & 29.3 & 26.7 & 25.4 \\
\hline & & $\mathrm{B}$ & 32.8 & 31.9 & 29.8 & 28.7 & 26.5 & 25.2 \\
\hline \multirow{3}{*}{ (19) } & & $\mathrm{R}$ & 35.7 & 34.8 & 31.8 & 30.9 & 28.7 & 27.5 \\
\hline & & $\mathrm{G}$ & 36.8 & 35.9 & 32.5 & 31.6 & 29.4 & 28.1 \\
\hline & & B & 36.3 & 35.4 & 32.6 & 31.6 & 29.6 & 28.2 \\
\hline \multirow{3}{*}{ (23) } & & $\mathrm{R}$ & 37.7 & 37.3 & 34.4 & 33.9 & 31.0 & 30.1 \\
\hline & & $\mathrm{G}$ & 39.3 & 38.7 & 35.5 & 34.8 & 31.9 & 30.8 \\
\hline & & B & 38.2 & 37.6 & 34.6 & 33.9 & 31.1 & 30.0 \\
\hline
\end{tabular}

Table 2. PSNR (dB) of demosaicking results using DLMMSE method [13] on the denoised CFA images. Computed excluding a 20-pixel border.
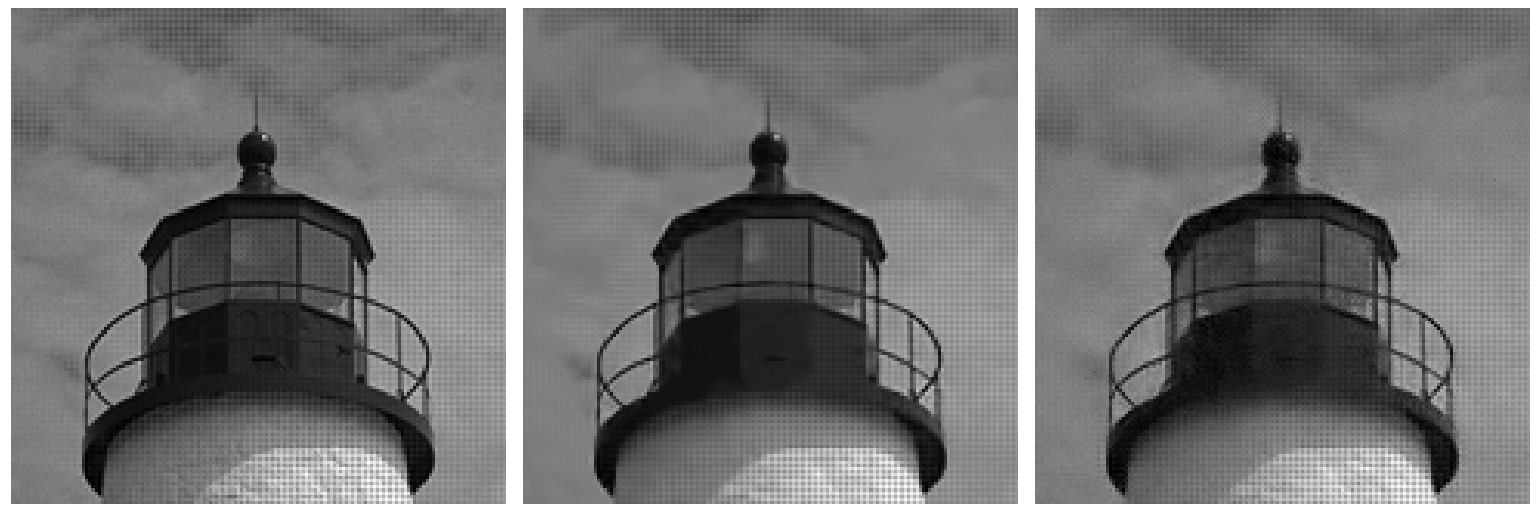

Figure 3. Lighthouse. From left to right: ground truth, denoised by proposed method, denoised by [12]. Gaussian noise $(\sigma=12 / 255)$.

[5] Hirakawa, K., and T. W. Parks, "Joint demosaicking and denoising," IEEE Trans. Image Process., vol. 15, no. 8, pp. 2146-2157, Aug. 2006.

[6] Katkovnik, V., A. Foi, K. Egiazarian, and J. Astola, "From local kernel to nonlocal multiple-model image denoising", Int. J. Computer Vision, July 2009.

[7] Koh, C.C., J. Mukherjee and S.K. Mitra, "New efficient methods of image compression in digital cameras with color filter array," IEEE Trans. Consumer
Electronics, vol. 49, no. 4, pp. 1448-1456, Nov. 2003.

[8] Muresan, D.D., and T.W. Parks, "Adaptive principal components and image denoising," Proc. ICIP 2003, vol. 1, pp 1101-1104, 14-17 Sep. 2003.

[9] Paliy, D., A.Foi, R. Bilcu, V. Katkovnik, "Denoising and Interpolation of Noisy Bayer Data with Adaptive Cross-Color Filters", SPIE-IS\&T Electronic Imag- 

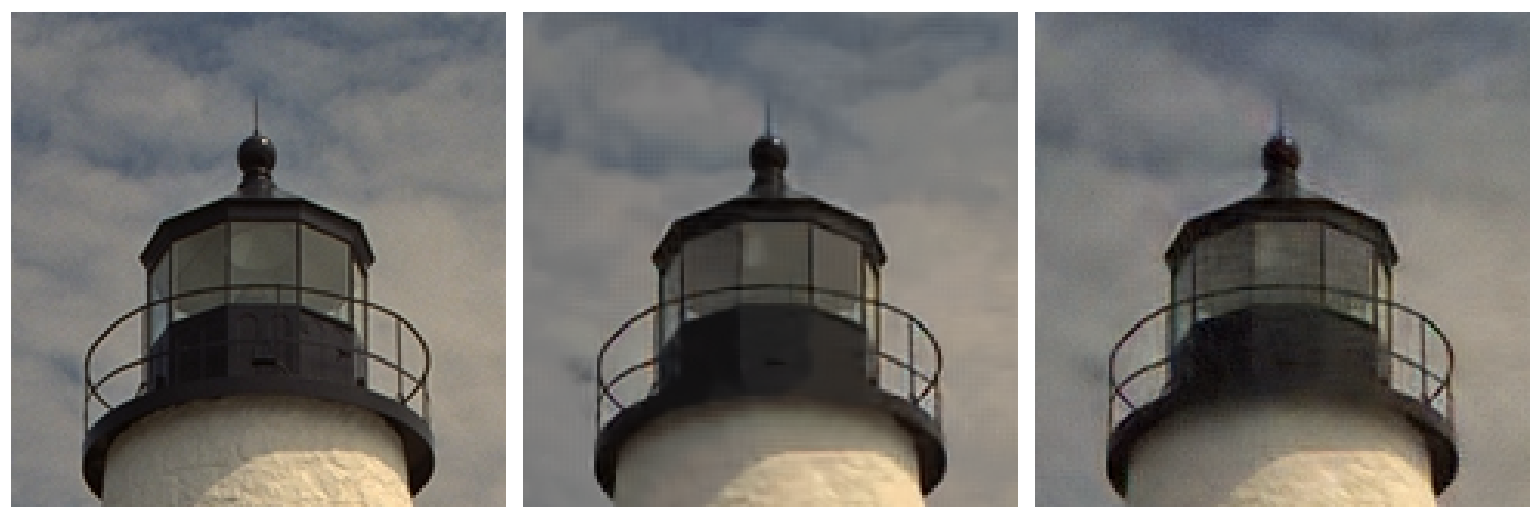

Figure 4. From left to right: ground truth, proposed denoising + interpolation [13], denoising [12] + interpolation [13]. Gaussian noise $(\sigma=12 / 255)$.
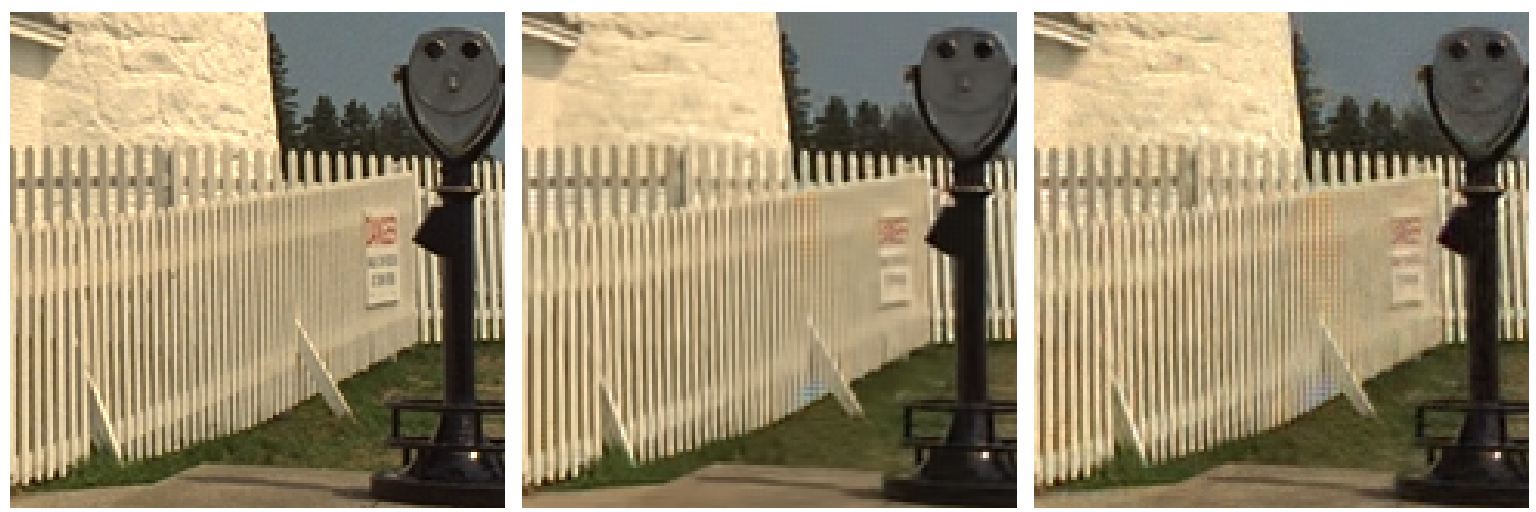

Figure 5. From left to right: ground truth, proposed denoising + interpolation [13], denoising [12] + interpolation [13]. Signal dependent noise $\left(a=0.004, b=0.02^{2}\right)$.

\begin{tabular}{|c|c|c|c|c|}
\hline & & & Proposed & [12] \\
\hline & \multicolumn{2}{|c|}{$\sigma(y)=\sqrt{a y+b}$} & \multicolumn{2}{|c|}{$a=0.004, b=0.02^{2}$} \\
\hline \multirow{3}{*}{$(07)$} & & $\mathrm{R}$ & 34.1 & 32.7 \\
\hline & & G & 34.9 & 33.5 \\
\hline & & B & 34.3 & 33.1 \\
\hline \multirow{3}{*}{$(08)$} & & $\mathrm{R}$ & 29.5 & 28.3 \\
\hline & & G & 30.4 & 29.2 \\
\hline & 目 & B & 29.7 & 28.5 \\
\hline \multirow{3}{*}{ (19) } & & $\mathrm{R}$ & 32.0 & 31.0 \\
\hline & & G & 32.6 & 31.7 \\
\hline & & B & 32.7 & 31.7 \\
\hline \multirow{3}{*}{ (23) } & & $\overline{\mathrm{R}}$ & 34.2 & 33.7 \\
\hline & & G & 35.4 & 34.7 \\
\hline & & B & 34.8 & 34.2 \\
\hline
\end{tabular}

Table 3. PSNR (dB) of denoised and demosaicked images corrupted by signal dependent noise. Computed excluding a 20-pixel border.

ing, Visual Communications and Image Processing 2008, vol. 6822, San Jose, CA, Jan. 2008.

[10] Paliy, D., V. Katkovnik, R. Bilcu, S. Alenius, K. Egiazarian, "Spatially Adaptive Color Filter Array Interpolation for Noiseless and Noisy Data", International Journal of Imaging Systems and Technol- ogy (IJISP), Special Issue on Applied Color Image Processing, vol. 17, no. 3, pp. 105-122, Oct. 2007.

[11] Trussell, H. J., and R. E. Hartwig, "Mathematics for demosaicking," IEEE Trans. Image Process., vol.11, issue 4, pp. 485-492, Apr. 2002.

[12] Zhang, L., R. Lukac, X. Wu and D. Zhang, "PCAbased Spatial Adaptive Denoising of CFA Images for Single-Sensor Digital Cameras," IEEE Trans. Image Process., vol. 18, no. 4, pp. 797-812, Apr. 2009.

[13] Zhang, L., and X. Wu, Color demosaicking via directional linear minimum mean square-error estimation, IEEE Trans. Image Process., vol 14, no. 12, pp. 2167-2178. 